\title{
Intravital imaging reveals systemic ezrin inhibition impedes cancer cell migration and lymph node metastasis in breast cancer
}

Abdi Ghaffari ${ }^{1,4^{*}+}$ (D), Victoria Hoskin ${ }^{1,4}$, Gulisa Turashvili ${ }^{1}$, Sonal Varma ${ }^{1}$, Jeff Mewburn ${ }^{4}$, Graeme Mullins ${ }^{1,4}$, Peter A. Greer ${ }^{1,4}$, Friedemann Kiefer ${ }^{2}$, Andrew G. Day ${ }^{5}$, Yolanda Madarnas ${ }^{3}$, Sandip SenGupta ${ }^{1}$ and Bruce E. Elliott ${ }^{1,4^{*}+}$

\begin{abstract}
Background: Limited understanding of the cancer biology of metastatic sites is a major factor contributing to poor outcomes in cancer patients. The regional lymph nodes are the most common site of metastasis in most solid cancers and their involvement is a strong predictor of relapse in breast cancer (BC). We have previously shown that ezrin, a cytoskeletal-membrane linker protein, is associated with lymphovascular invasion and promotes metastatic progression in BC. However, the efficacy of pharmacological inhibition of ezrin in blocking cancer cell migration and metastasis remains unexplored in $\mathrm{BC}$.

Methods: We quantified ezrin expression in a BC tissue microarray $(n=347)$ to assess its correlation with risk of relapse. Next, we developed a quantitative intravital microscopy (qIVM) approach, using a syngeneic lymphatic reporter mouse tumor model, to investigate the effect of systemic ezrin inhibition on cancer cell migration and metastasis.

Results: We show that ezrin is expressed at significantly higher levels in lymph node metastases compared to matched primary tumors, and that a high tumor ezrin level is associated with increased risk of relapse in BC patients with regional disease. Using qIVM, we observe a subset of cancer cells that retain their invasive and migratory phenotype at the tumordraining lymph node. We further show that systemic inhibition of ezrin, using a small molecule compound (NSC668394), impedes the migration of cancer cells in vivo. Furthermore, systemic ezrin inhibition leads to reductions in metastatic burden at the distal axillary lymph node and lungs.
\end{abstract}

Conclusions: Our findings demonstrate that the tumor ezrin level act as an independent biomarker in predicting relapse and provide a rationale for therapeutic targeting of ezrin to reduce the metastatic capacity of cancer cells in high-risk BC patients with elevated ezrin expression.

Keywords: Ezrin, Quantitative intravital imaging, Cell migration, Lymph node metastasis, Biomarker, Metastatic disease

\footnotetext{
*Correspondence: ghaffari@queensu.ca; elliottb@queensu.ca

Abdi Ghaffari and Victoria Hoskin are co-first authors.

${ }^{+}$Abdi Ghaffari and Victoria Hoskin contributed equally to this work.

'Department of Pathology and Molecular Medicine, Queen's University,

Kingston, Canada

Full list of author information is available at the end of the article
}

(c) The Author(s). 2019 Open Access This article is distributed under the terms of the Creative Commons Attribution 4.0 International License (http://creativecommons.org/licenses/by/4.0/), which permits unrestricted use, distribution, and reproduction in any medium, provided you give appropriate credit to the original author(s) and the source, provide a link to the Creative Commons license, and indicate if changes were made. The Creative Commons Public Domain Dedication waiver (http://creativecommons.org/publicdomain/zero/1.0/) applies to the data made available in this article, unless otherwise stated. 


\section{Background}

Cancer is not only a disease of uncontrolled growth, but in its most aggressive form it is also a disease of uncontrolled cell migration. Ezrin, a member of the ezrin-radixin-moesin (ERM) family of actin cytoskeleton-plasma membrane linker proteins, is involved in multiple aspects of cancer cell migration and its overexpression has been associated with poor prognosis in a variety of solid tumors [1]. Ezrin plays a critical role in epithelial morphology, adhesion, and migration - all key events that contribute to the invasive phenotype of cancer cells during tumor progression [2]. It is therefore not surprising that genetic ablation of ezrin or mutation of its key binding sites has been shown to impair metastatic progression in experimental models of a variety of solid cancers [3].

Lymph nodes (LNs) are the most common sites of solid tumor metastases, and spread of cancer cells to regional LNs is one of the strongest predictors of risk of relapse in $\mathrm{BC}$ patients [4]. In $\mathrm{BC}$, there is extensive evidence for preferential dissemination of cancer cells via the lymphatic rather than blood vascular route [5]. Furthermore, the addition of regional nodal irradiation to conventional treatment, after mastectomy or breast-conserving surgery in node-positive patients, reduces the rate of locoregional and systemic recurrence $[6,7]$. Despite this clinical importance, and partly due to a lack of appropriate single-cell resolution imaging models of LN metastasis, we have a very limited understanding of the fate of cancer cells within LNs and of the efficacy of therapeutic targeting of prometastatic molecules to prevent further metastatic spread of cancer cells beyond regional LNs. We have previously shown that tumor ezrin levels correlate with lymphovascular invasion in a locally accrued BC cohort and that ezrin acts cooperatively with Src in regulating tumor lymphangiogenesis [8]. Clinically, ezrin overexpression has also been reported to correlate with the presence of lymph node metastasis in breast cancer [9], pancreatic cancer [10], and nonsmall-cell lung cancer [11]. Together, these findings prompted us to examine whether pharmacological inhibition of ezrin could have therapeutic benefits by suppressing the spread of highly metastatic cancer cells from lymph node micrometastases.

Ezrin's interactions are dependent on conformational activation of the molecule. In its inactive state, ezrin binding sites are masked by intramolecular interaction between its N-terminal 4.1/ezrin/radixin/moesin (FERM) domain and the C-terminus. Upon phosphorylation of a conserved $\mathrm{C}$-terminal threonine residue ( $\mathrm{T} 567$ ) by protein kinase $\mathrm{C}$ or Rho kinase and subsequent binding with membrane-associated phosphatidylinositol 4,5-biphosphate (PIP2), the intramolecular masking of its binding sites is removed and interaction with binding partners can take place [2]. Bulut and colleagues have recently discovered several small molecule inhibitors with high binding affinity to ezrin and their ability to block T567 phosphorylation [12-14]. These authors demonstrated a reduction in the invasive phenotype of cancer cells and inhibition of lung metastases in mice treated with ezrin inhibitors in osteosarcoma experimental models [12].

In this study, we have shown that elevated tumor ezrin protein expression is associated with increased risk of relapse in node-positive and high-risk node-negative $\mathrm{BC}$ patients. We developed a novel qIVM approach using tumor-bearing lymphatic reporter mice to track metastatic cancer cell migration in vivo in real time. We observed a significant reduction in migration and invasion capacity of cancer cells in tumor-draining inguinal lymph node metastases following pharmacological inhibition of ezrin, with a concomitant decrease in metastatic burden in the draining axillary node.

\section{Methods}

Breast cancer patient cohort and tissue microarray

A tissue microarray (TMA) $(n=347)$ was constructed from unselected archival (formalin-fixed paraffin-embedded) breast tumor specimens from consenting patients treated at the Cancer Centre of Southeastern Ontario at Kingston General Hospital (SEOBC cohort) between 1996 and 2007 $(n=450)$. A summary of clinicopathological data is presented in Additional file 1: Figure S1A. Written informed consent was obtained from all patients and the studies were conducted in accordance with the Queen's University Research Ethics Board consent guidelines. Patients with previous history of cancer, bilateral disease, or neoadjuvant chemotherapy were excluded. Archival reduction mammoplasties from consenting patients were included as nonmalignant controls. Each sample was represented by triplicate cores $(0.6 \mathrm{~mm})$ from the tumor and adjacent benign tissue, carefully annotated by two pathologists (SV, SSG). Immunohistochemistry (IHC) staining of ER, PR, HER2, Ki-67, EGFR, and CK5/6 was performed at the Centre for Translational and Applied Genomics (BC Cancer Agency, Vancouver, BC, Canada) and scored visually by a pathologist (GT). High-risk node-negative BC is defined as patients with tumor size larger than $1.0 \mathrm{~cm}$ and one or more of the following parameters: tumor grade $\geq 3$, negative ER status, and positive lymphovascular invasion (LVI). Ezrin gene expression data were obtained from 844 breast cancer patients enrolled in The Cancer Genome Atlas (TCGA) study as described previously and based upon data generated by the TCGA Research Network (http//cancergenome.nih.gov) [15]. Normalized RNA-Seq data (version 2, level 3) were used to analyze ezrin gene expression in benign and breast tumor tissues.

\section{Immunohistochemistry}

IHC was performed as previously described [15]. Biomarker (ezrin) staining and analysis on our BC cohort 
was performed according to REMARK guidelines [16]. In brief, freshly cut $5 \mu \mathrm{m}$ thick TMA, whole tissue sections, or mouse tumour tissue were stained with antibodies against ezrin (Sigma-Aldrich, Oakville, ON; cat\# E8897, clone 3C12), and AE1/AE3 cytokeratin (Santa Cruz Biotechnologist, Dallas, Texas; cat\# sc-81,714) antibodies using the automated Ventana Discovery XT staining system (Ventana Medical Systems, Tucson AZ) and EDTA buffer for antigen retrieval process $\left(\mathrm{pH} 8.0,100^{\circ} \mathrm{C}\right)$. Ezrin-expressing and ezrin-deficient cell pellets were included in the TMA as positive and negative controls. Technical reproducibility of ezrin stain was assessed by comparing replicate staining of serial sections from whole tumour tissue as well as a test BC TMA. Tumour tissues harvested from mice were processed for IHC staining by Ventana system using lyve-1 (Millipore Sigma, Etobicoke, ON, cat\# AB2988) and CD31 (Santa Cruz, cat\# sc-1506) antibodies as previously described [8]. Number of lymphatic (lyve- $1^{\text {high }}$, CD $31^{\text {low }}$ ) and blood (lyve- $1^{\text {low }}, \mathrm{CD} 31^{\text {high }}$ ) vessels were quantified (manual count by students blinded to the study) in at least 5 random fields of view (200X magnification) in peritumoral regions.

\section{TMA automated scoring}

TMA slides were scanned by ScanScope (Aperio Technologies, Vista, CA, USA) after IHC to obtain the digital images. Automated scoring of ezrin IHC stains was performed as described previously [15]. In brief, the HALO (Indica Labs Inc., Corrales, NM, USA) algorithm was optimized, under pathologist supervision, using a cytoplasmic/membrane script, which gates hematoxylin-stained tumor nuclei based on size, shape, compactness, and roundness. This, in combination with manual annotations and a classifier that recognized the stromal pattern, allowed for scoring ezrin stains at a single-cell resolution in only tumor regions of TMA cores. The HALO output summarizes the percentage of tumor cells in each core that stained negative, weak, moderate, and strong for ezrin expression. A histopathology score (H-score) was then calculated for each core by multiplying percent positive cell at each staining intensity (a value from 0-300) and expressed as the average of 3 cores per tumor/patient. Cores with less of 50 tumor cells were excluded from the analysis.

\section{Cell lines and plasmids}

EO771 medullary breast adenocarcinoma cells were purchased in 2014 (cat\# 940001; CH3 BioSystems, Amherst, NY, USA), and were originally isolated by Dr FM Sirotnak (Memorial Sloan-Kettering Cancer Centre, New York, NY, USA) from a spontaneous cancer in C57BL/6 mice [17]. Orthotopic and subcutaneous syngeneic tumors derived from the EO771 cell line were described previously [18, 19]. EO771 cells were cultured in DMEM with 10\% FBS (Sigma-Aldrich) and 1\% glutamine supplement. EO771 cells were transduced with GFP-expressing ecotropic lentivirus containing pWPXLD plasmid using Polyjet (Froggabio, North York, ON Canada) according to the manufacturer's instructions. A highly metastatic variant of the GFP-EO771 cell line was selected as described previously with some modifications [20]. GFP-EO771 cells $\left(5 \times 10^{5}\right.$ cells) suspended in Matrigel/PBS (50:50) were injected into the mammary fat pad (MFP) of female C57BL/6 mice using a Hamilton syringe. Four weeks later, mice were dissected and examined for established lung metastases using a dissecting microscope. A single lung nodule was dissected, minced, and engrafted into the MFP of a female C57BL/6 mouse using a 16-gauge needle. This process was repeated three times, with the final metastatic isolates cultured in growth media for the generation of a stable lung metastatic variant (LMV) of GFP-EO771 cell line $\left(\mathrm{EO} 771^{\mathrm{LMV}}\right)$. All animal procedures were carried out according to the guidelines of the Canadian Council on Animal Care with the approval of the Queen's University Animal Care Committee. MDA-MB-231 human BC cells were obtained from Dr P Siegel in 2007 (McGill University, Montreal, QC, USA) and transfected with human Ezrin shRNA or empty vector control pLKO.1 lentiviral vector as described previously [20]. All cultured cell lines were used before passage 10 from the original frozen stocks and routinely tested for mycoplasma (Lookout Mycoplasma PCR Detection Kit, Sigma Aldrich) and found to be free of contamination.

\section{Immunoblotting}

Whole-cell lysates were prepared as described previously [20]. Total protein concentrations were determined using the DC Protein Assay (Bio-Rad, Mississauga, ON, Canada). Lysate proteins $(10-20 \mu \mathrm{g})$ were separated by SDS-PAGE, transferred to $0.45-\mu \mathrm{m}$ PVDF membranes (EMD Millipore, Etobicoke, ON, Canada), blocked in 5\% nonfat dry milk in $1 \times$ Tris-buffered saline $/ 0.1 \%$ Tween-20, and then probed with rabbit anti-ezrin (cat\# 3145; Cell Signaling), anti-phospho-threonine Ezrin/Radixin/Moesin (pTERM, cat\# 3149; Cell Signaling), and anti- $\gamma$-tubulin (cat\# T5326; Sigma-Aldrich).

\section{Real-time in-vitro cell migration assay}

GFP-EO771 ${ }^{\text {LMV }}$ cells were seeded $\left(2 \times 10^{4}\right.$ cells $)$ sparsely onto collagen-coated four-well $\mu$-slides (Ibidi, Madison, WI, USA) in DMEM plus 10\% FBS and $20 \mathrm{mM}$ HEPES, and incubated at $37^{\circ} \mathrm{C}$ for $2 \mathrm{~h}$. Cells were then imaged every $10 \mathrm{~min}$ for $15 \mathrm{~h}$ using a $10 \times$ objective on an inverted Quorum WaveFX-X1 Spinning Disk confocal microscope equipped with a closed chamber at $37^{\circ} \mathrm{C}$ and $5 \% \mathrm{CO}_{2}$. Individual cells were tracked using MetaMorph software by two observers blinded to the study. Cell tracking and directional migration of cell migration were analyzed using the open source DiPer program 
[21] and Excel. A minimum of 30 cells were tracked per experiment.

\section{Intravital imaging of lymph node metastasis}

All animal procedures were carried out according to the guidelines of the Canadian Council on Animal Care with the approval of the Queen's University Animal Care Committee. Female prox1-mOrange2-pA-BAC lymphatic reporter mice (8-10 weeks old), developed by Dr F Kiefer and colleagues [22], were injected subcutaneously into the left flank with GFP-EO771 ${ }^{\text {LMV }}$ cells $\left(1 \times 10^{5}\right.$ cells $)$. When tumors reached $400 \mathrm{~mm}^{3}$ ( $\sim 20$ days), mice were anesthetized with ketamine $(200 \mathrm{mg} / \mathrm{kg})$ and xylazine $(10 \mathrm{mg} / \mathrm{kg})$ and a jugular vein catheter was inserted for intravenous delivery of anesthetic during the procedure. Skin flap surgery was performed to expose the inguinal LN by careful removal of adipose and connective tissue. The skin flap was then stabilized on a heated motorized microscope stage using holding clamps, a $2 \mathrm{~cm} \times 2 \mathrm{~cm}$ piece of foam, and surgical tape to avoid tissue drift and compression during imaging. Intravital imaging was performed on a Confocal Quorum WaveFX-X1 spinning-disk microscope (Quorum Technologies, Guelph, Canada) equipped with a Hamamatsu EMCCD camera (Hamamatsu, Japan), based on an imaging system previously described by Dr Paul Kubes' group [23], which allowed for simultaneous visualization of GFP-expressing cancer cells (Ex $491 \mathrm{~nm}$ ), prox1-mOra nge2-expressing lymphatic endothelial cells (LECs) (Ex $561 \mathrm{~nm}$ ), and eFluor660-CD3-labeled T cells (Ex $642 \mathrm{~nm}$; eBioscience, San Diego, CA, USA). To visualize blood vasculature, Alexa647-CD31 antibody (BioLegend, San Diego, CA, USA) was injected intravenously $(200 \mu \mathrm{l})$ using the catheter prior to imaging. To track cell motility in TDLN or trafficking within lymphatic vessels, 4D images were acquired with a $20 \times$ objective every $30-60 \mathrm{~s}$ for up to $2.5 \mathrm{~h}$ with $2-\mu \mathrm{m}$ z-stacks spanning $4-20 \mu \mathrm{m}$ from the focal plane and a maximum depth of $100 \mu \mathrm{m}$ into the inguinal LN cortex. Images were analyzed in Metamorph software (Molecular Devices, Sunnyvale, CA, USA) with minimal processing to reduce noise and adjust brightness/contrast. GFP-expressing cancer cells were manually tracked in Metamorph Software by two students blinded to the study. Cell track data were graphed in Microsoft Excel using the DiPer macro method described previously [21]. The number of fields per LN, number of mice, and number of experiments are indicated in the appropriate figure legends.

\section{Assessment of lung metastasis in EO771 orthotopic tumor model}

GFP-EO771 ${ }^{\text {LMV }}$ cells $\left(5 \times 10^{5}\right.$ cells $)$ suspended in Matrigel/PBS (50:50) were injected into the (fourth) mammary fat pad of female C57BL/6 mice using a Hamilton syringe. When tumors reach a palpable size $\left(\sim 100 \mathrm{~mm}^{3}\right)$, mice received daily treatment of ezrin inhibitor NSC668394 (0.5 $\mathrm{mg} / \mathrm{kg}$, i.p.) or vehicle ( $0.01 \% \mathrm{DMSO} / \mathrm{PBS})$ until tumors reached $\sim 1 \mathrm{~cm}$ in diameter ( day 20$)$. At this point, primary tumors $(\sim 1 \mathrm{~cm}$ diameter) were surgically removed and mice were allowed to recover. Lung metastases were allowed to expand for 1 week more prior to harvesting and assessment of total lung tumor nodules by fluorescence imaging.

\section{Statistical analysis}

All statistical analyses were performed using SPSS or GraphPad Prism software, unless otherwise indicated. Data are presented as mean $\pm \mathrm{SD}$ and $p<0.05$ was considered significant. Specific statistical tests are described in the figure legends. In brief, the $p$ values were calculated by Student's $t$ test or Mann-Whitney $U$ test between two means and by Kruskal-Wallis test followed by Dunnett's multiple comparison tests for three or more means. The log-rank test was used to assess statistical significance between Kaplan-Meier disease-free survival curves. Statistical analyses of clinical outcome were performed under supervision of the team's biostatistician (AGD).

\section{Results \\ High tumor ezrin levels correlate with increased risk of relapse in invasive $\mathrm{BC}$}

To assess the association between ezrin and risk of metastasis in $\mathrm{BC}$, we quantified ezrin protein expression in primary tumors ( $n=347$, Additional file 1 : Figure S1A) and a subset of matched benign ductal tissues $(n=90)$ in TMA cores, using immunohistochemistry (IHC) and HALO automated quantitative image analysis (Fig. 1a). Ezrin scores show strong core-to-core reproducibility in our TMA (Additional file 1: Figure S1B). Total ezrin protein levels showed an average of 4-fold increase in breast tumors compared to matched benign ductal tissues (Fig. 1b). Ezrin gene $(E Z R)$ expression was also significantly elevated in breast tumor compared with benign tissues (Fig. 1b). As ezrin plays a critical role in cancer cell invasion, we next explored its prognostic potential in patients with higher risk of metastatic disease. In multivariate survival analyses, elevated expression of ezrin in primary tumor (median used as cutoff) was associated with increased risk of relapse (DFS; HR = 2.0 (95\% CI 1.0-4.0), $p=0.04$ ) and mortality (OS; HR $=2.5$ (95\% CI 1.2-5.2), $p=0.01$ ) in node-positive and high-risk node-negative patients. Survival analyses of unselected BC patients also suggested an association between elevated tumor ezrin levels and increased risk of relapse (Additional file 1: Figure S1C). Tumor ezrin levels were not prognostic in node-negative $\mathrm{BC}$ (Additional file 1: Figure S1C). Analysis of a small subset of matched primary tumor and LN-positive lesions from the same patient revealed significantly higher ezrin expression in LN metastases (Fig. 1e). Interestingly, higher levels of phospho-ezrin (pTERM, activated ezrin) were also observed in the 


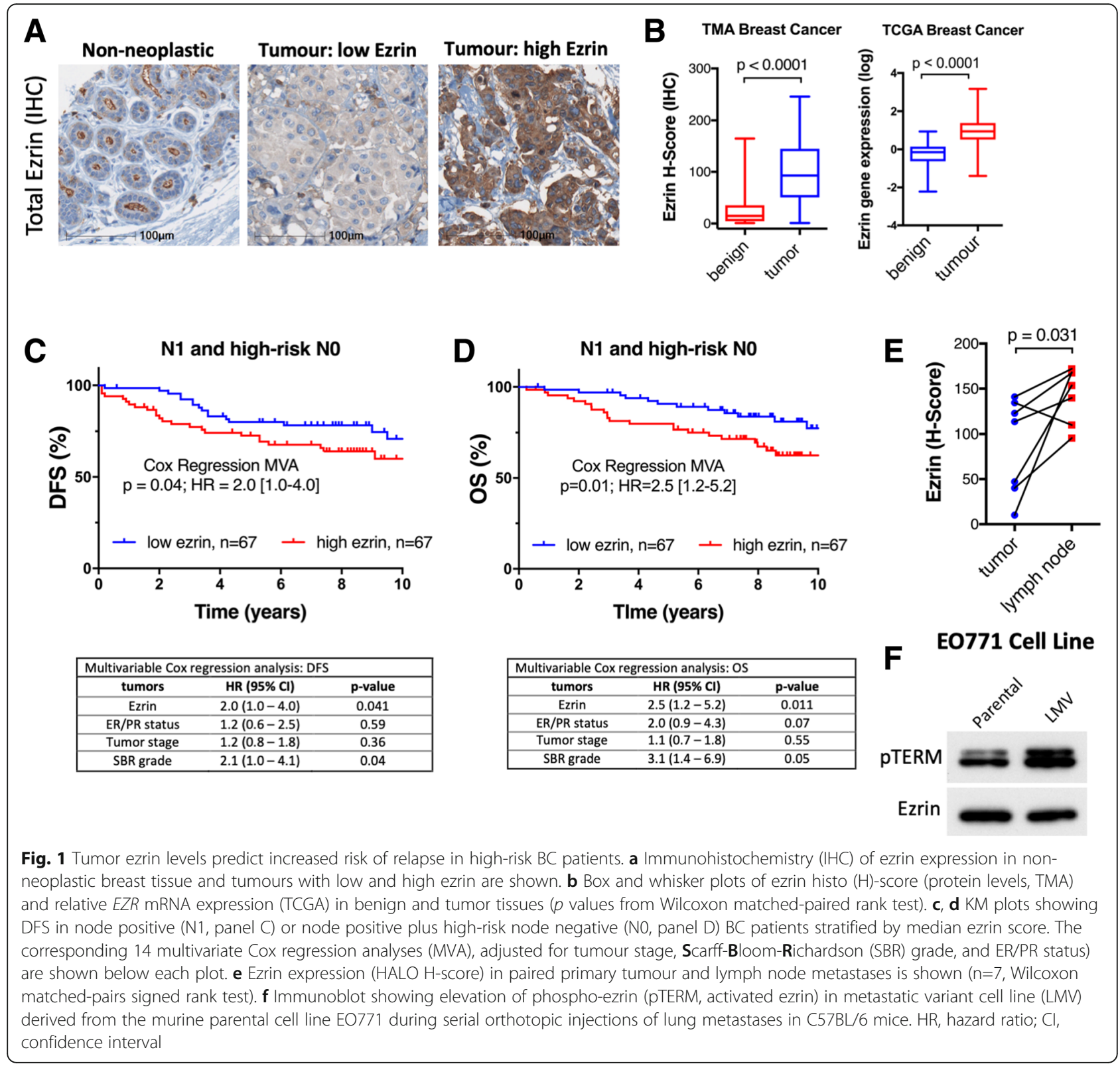

metastatic variant cell line compared with the parental EO771 cells (Fig. 1f). These findings further support a role for ezrin in cancer cell invasion and as a potential marker of cancer progression and relapse in high-risk BC.

Development of an intravital imaging model to study the effects of ezrin-targeted therapy on cancer cell migration in LN metastases

The association between elevated ezrin expression and increased risk of metastases in node-positive $\mathrm{BC}$ prompted us to investigate the effect of pharmacological inhibition of ezrin to restrain cancer cell migration in vivo. We generated a highly metastatic cancer cell line (GFP-EO771 ${ }^{\mathrm{LMV}}$ ) from lung metastatic nodules following engraftment of the GFP-EO771 murine mammary carcinoma cells into wild-type C57BL/6 mice. Next, we developed a qIVM model to directly visualize metastatic cancer cell migration within the tumor-draining inguinal $\mathrm{LN}$ in syngeneic tumors engrafted into lymphatic reporter prox1-mOrange 2 mice [22] (Additional file 2: Figure S2). As orthotopic mammary fat pad tumors commonly engulf the entire inguinal node in mice, we used a subcutaneous model for optimal intravital imaging of LN metastases. We observed LN metastasis in all tumor-bearing mice in our model and metastatic lesions were primarily found in the cortex region near the subcapsular sinus (SCS) of the inguinal LN (Fig. 2a). To 


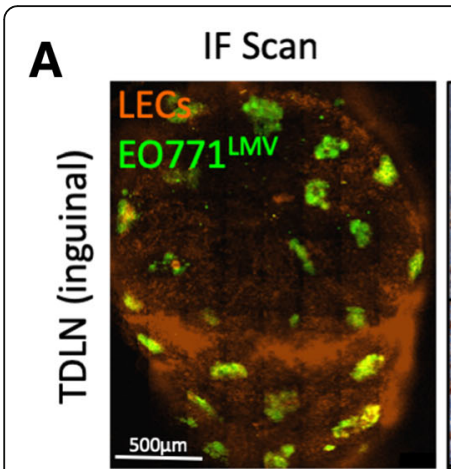

C

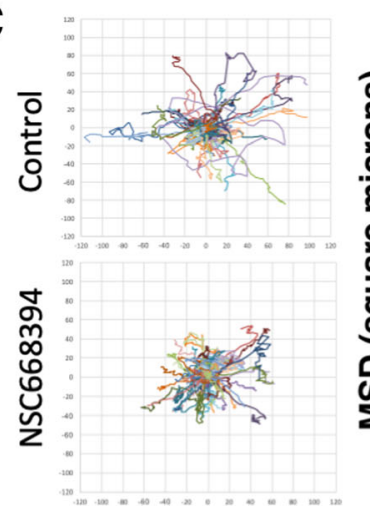

pan CK (IHC)

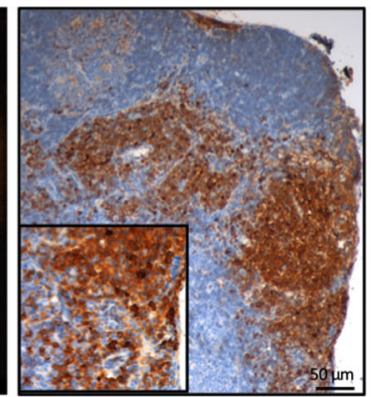

E0771-LMV cell line

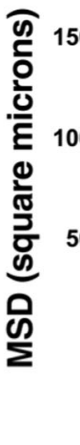

B

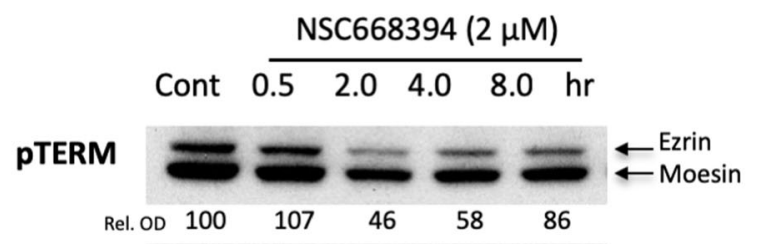

Ezrin

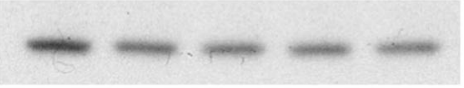

D

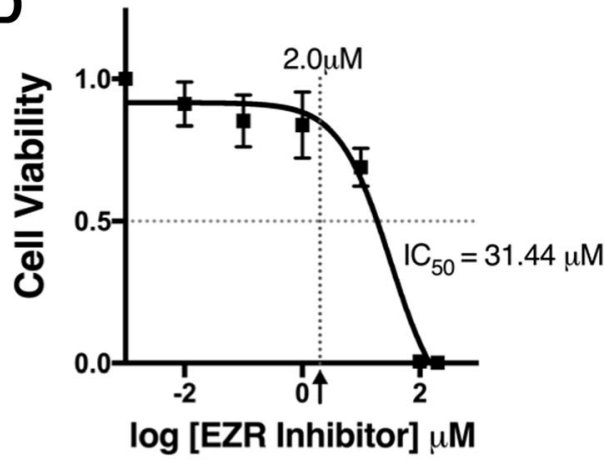

Fig. 2 GFP-EO771 ${ }^{\mathrm{LMV}}$ tumors develop spontaneous LN metastases in subcutaneous and orthotopic models. a Whole tissue confocal scan of inguinal TDLN (left, scale bar $500 \mu \mathrm{m}$ ) and cytokeratin stain (right) confirming presence of GFP-EO771 ${ }^{\text {LMV }}$ metastases. b Immunoblot analysis of GFP-EO771 $1^{\text {LMV }}$ cells treated with NSC668394 ezrin inhibitor $(2 \mu \mathrm{M})$ in vitro show reduction in ezrin pT567 (upper pTERM band). The percent ratio of phospho-ezrin to total protein normalized to control (relative optical density (Rel. OD)) shown under each band (mean of $n=2$ assays). c Migration of GFP-EO771 $1^{\mathrm{LMV}}$ cells in response to ezrin inhibitor in vitro analyzed by time-lapse microscopy for up to $18 \mathrm{~h}$ (see Additional file 3: video 1). Cell trajectories (left, minimum of 30 cells/group, pooled from three independent assays) were used to plot mean square displacement curves (right panel) using DiPer software $(p<0.0001$, Wilcoxon matched-paired signed rank test). d Cell viability analysis shows the half maximal inhibitory concentration IC 50 in NSC668394 treatment of GFP-EO771 ${ }^{\text {LMV }}$ cells. Th arrow points to $2.0 \mu \mathrm{M}$ value on the $x$ axis (mean of three independent assays).

target ezrin activity in vivo, we used a novel small molecule inhibitor (NSC668394) described previously by Bulut et al. in an osteosarcoma model [12]. GFP-EO771 ${ }^{\mathrm{LMV}}$ cells express ezrin and display marked reductions in phospho-ezrin pT567 level (Fig. 2b) and in-vitro migration capacity (Fig. 2c, Additional file 3: video 1 and Additional file 4: video 2) when treated with NSC668394 at concentrations $(2.0 \mu \mathrm{M})$ well below the $\mathrm{IC}_{50}$ value (Fig. $2 \mathrm{~d}$ ). Migration efficiency of ezrin-deficient MDA-MB-231 cells treated with NSC668394 was not affected in comparison to their wild-type counterpart, further supporting the specificity of the inhibitor (Additional file 5: Figure S3A, B). Ezrin inhibitor had no significant effect on the rate of mitosis in MDA-MB-231 cells (Additional file 5: Figure S3C).

\section{Systemic treatment with an ezrin inhibitor reduces migration of cancer cells in vivo}

Using our qIVM lymphatic reporter model, we next examined the effect of systemic ezrin inhibition on cell migration dynamics within LN metastases. On day 20 post tumor injection, mice received two sequential doses of NSC668394 $(0.5 \mathrm{mg} / \mathrm{kg}$, i.p.) or vehicle $(0.01 \% \mathrm{DMSO} / \mathrm{PBS})$ alone at 24 $\mathrm{h}$ and $6 \mathrm{~h}$ prior to imaging (Additional file 2: Figure S2). We captured simultaneous time-lapse images from multiple sites in each ipsilateral inguinal $\mathrm{LN}$ for up to $2.5 \mathrm{~h}$ (Fig. 3a, Additional file 6: video 3 and Additional file 7: video 4). The trajectories (normalized to the origin) of all motile cells were tracked as shown in Fig. 3b. On average, about $8 \%$ of cancer cells per field of view were actively migrating within TDLNs in the untreated group, and this percentage decreased significantly in NSC668394-treated mice (Fig. 3c). The slope of the mean displacement curve was significantly reduced in motile cancer cells in mice treated with NSC668394 compared to cells in untreated mice, suggesting a reduction in their migration efficiency (Fig. 3d). Motile cells in untreated TDLNs displayed short bursts of solitary amoeboid-like movements with speeds reaching $2.5 \mu \mathrm{m} / \mathrm{min}$ and a mean velocity of $1.44 \pm 0.4 \mu \mathrm{m} / \mathrm{min}$. 


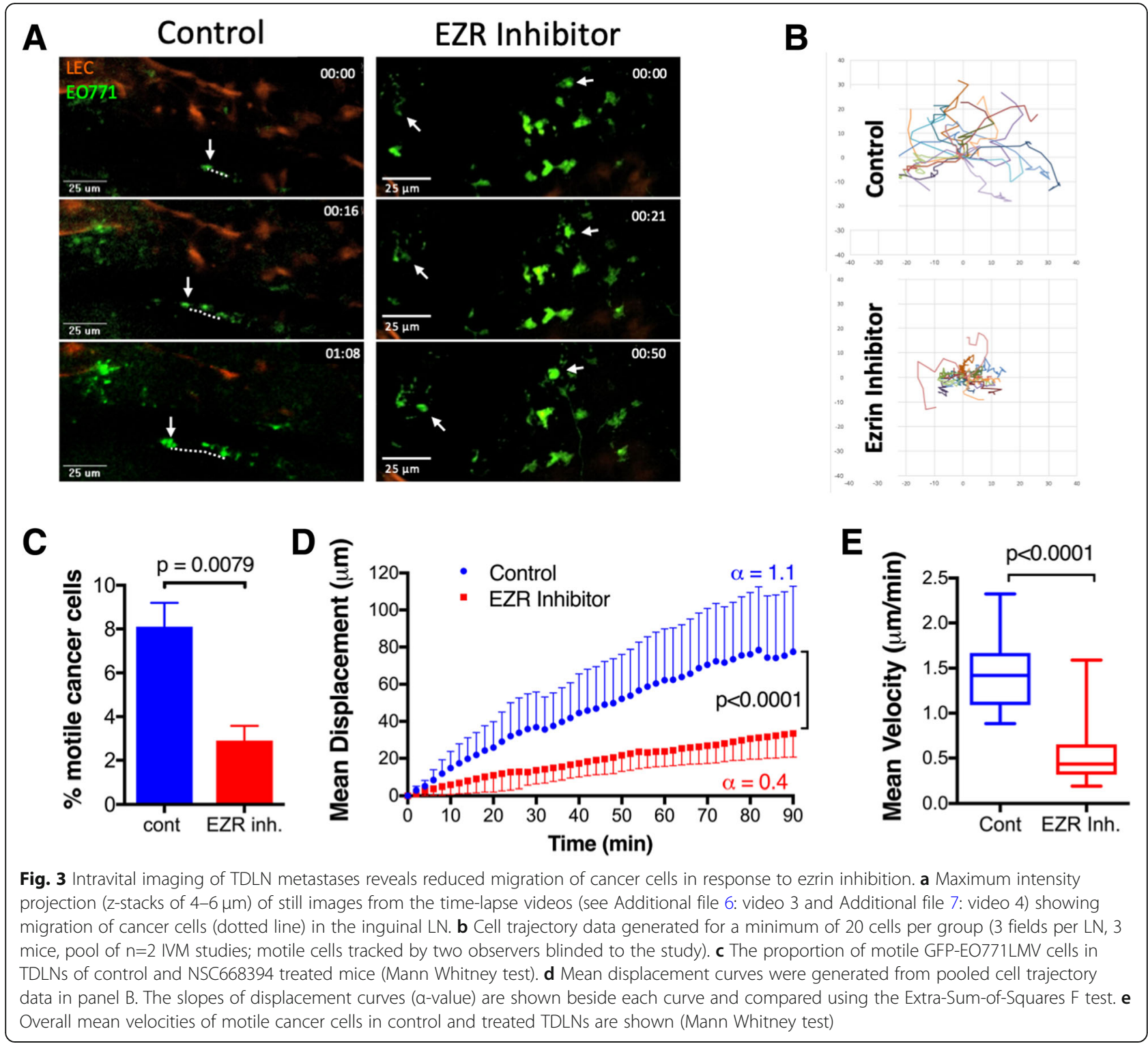

Ezrin inhibition did not affect the single-cell mode of motility, but cells displayed a more constrained movement with reduced top speed $(1.5 \mu \mathrm{m} / \mathrm{min})$ and a mean velocity of $0.56 \pm 0.3 \mu \mathrm{m} / \mathrm{min}$ (Fig. 3d, e). Although EO771 cells are capable of a mesenchymal-like (spindle-shape) mode of migration (see Additional file 3: video 1 and Additional file 4: video 2), motile cancer cells in TDLN displayed only a single-cell amoeboid-like (rounded) mode of migration, with no evidence of collective or mesenchymal-like motility (data not shown).

Ezrin inhibition reduces metastatic burden in the axillary $\mathrm{LN}$ and lungs

We next examined whether treatment with the ezrin inhibitor would reduce tumor colonization and metastatic burden in the distal node (axillary) and lungs in an orthotopic mammary fat pad tumor engraftment model. Mice were injected daily with NSC668394 (0.5 $\mathrm{mg} / \mathrm{kg}$, i.p.) or DMSO/PBS (control) for 7 days after GFP-EO771 ${ }^{\text {LMV }}$ tumors reached a palpable size $(\sim$ day $13)$. When tumors in the control group reached $\sim 500$ $\mathrm{mm}^{3}$, we harvested axillary nodes and lungs and scanned them by confocal fluorescent microscope to quantify the metastatic load (Fig. 4a). Ezrin inhibition reduced the number of tumor colonies per node and metastatic burden by 50\% (Fig. 4b) and 70\% (Fig. 4c), respectively. Minimal metastases were observed in contralateral axillary nodes (Fig. 4b, c). Mice in the ezrin inhibitor-treated group also exhibited significantly lower numbers of total lung metastases compared to the control group (Fig. 4d). Assessment of lymphatic and blood vascular density of primary tumors did not reveal a significant 


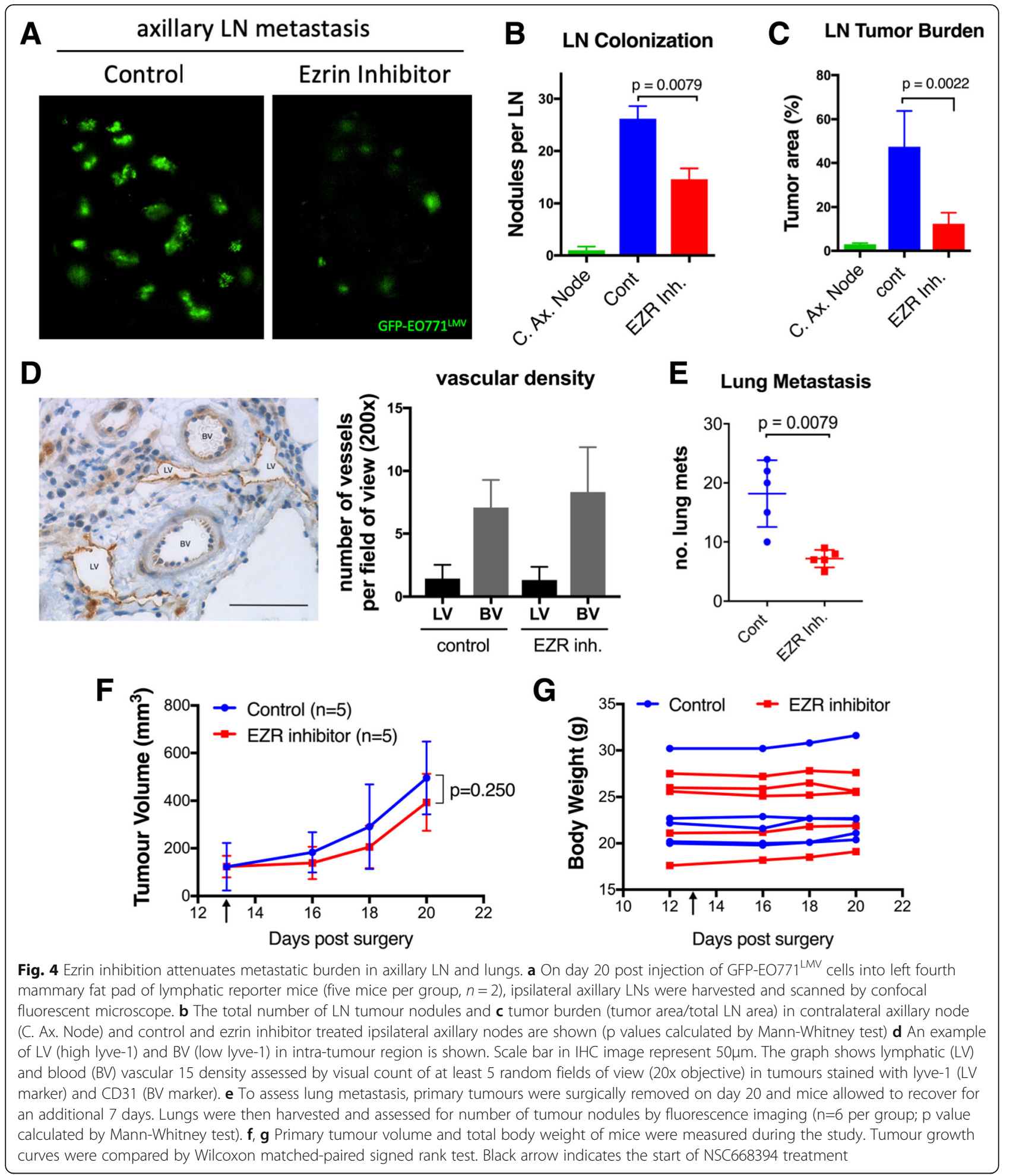

difference between control and ezrin inhibitor-treated groups (Fig. 4e). The primary tumors were considered size-matched as we did not observe a significant difference in primary tumor size between the two treatment groups (Fig. 4f). Mice treated with NSC668394 showed no significant difference in body weight (Fig. 4g), food intake, or physical activity (data not shown). Knockdown of ezrin in an MDA-MB-231 orthotopic tumor model also resulted in significant reductions in axillary LN and lung metastases (Additional file 8: Figure S4). 


\section{Discussion}

In the present study, we have identified ezrin as an independent prognostic marker for relapse in node-positive and high-risk node-negative BC patients. Together, with elevated ezrin expression in LN metastases compared to matched primary tumors, these data support our contention that ezrin overexpression confers a metastatic advantage to $\mathrm{BC}$ cells during tumor progression. To study the effect of ezrin inhibition on cancer cells in vivo, we have developed a novel intravital model using a lymphatic reporter mouse to directly track cancer cell migration within lymph node metastatic nodules. A growing body of evidence implicates ezrin as a key promoter of the metastatic process in solid tumors (reviewed in [3]). Ezrin plays a critical role in maintaining epithelial integrity and in focal adhesion and invadopodia turnover, both key processes in metastatic progression of cancer cells [2, 20, 24, 25]. Data from our laboratory and other groups have shown that ezrin overexpression in breast cancer cells increases cell scattering and invasion [26, 27], whereas knockdown or mutational inactivation of ezrin reduces cancer cell migration and diminishes the metastatic potential of cancer cells [20, 28-30]. The data presented here, to our knowledge, are the first to show that pharmacological inhibition of ezrin can effectively impede migratory capacity of cancer cells in vivo and reduce metastatic burden in LNs and lungs in BC models.

Metastasis is a complex process involving tumor cell motility, intravasation, circulation in the blood or lymph system, extravasation, and growth in distant sites [31]. The increase in metastatic cancer cell motility and invasiveness is a prerequisite for cancer progression and metastasis. Therefore, one can predict that a decrease in cancer cell motility in ezrin inhibitor-treated mice would contribute to the reductions in LN and lung metastatic burden observed in this study. However, it is important to acknowledge the existence of non-ezrin-dependent mechanisms of cell migration that may play an important role in metastatic progression. The elongated mode of cell motility (or mesenchymal-like motility) has been shown to be associated with Rac-dependent F-actin-rich protrusions independent of ezrin function [32]. We cannot exclude the contribution of alternate modes of cancer cell motility in our model. In fact, we do not observe complete inhibition of cell migration in vitro or full block of distant metastases in vivo following ezrin knockdown or inhibition, suggesting that non-ezrin-dependent pathways also play a role in our model. Furthermore, clinical evidence suggests that increased lymphatic vascular density in primary tumors is associated with increased lymphatic metastasis and poor outcome in $\mathrm{BC}$ [5]. We have previously shown that ezrin knockdown in breast cancer cells leads to reduced lymphangiogenic activity in a Matrigel plug xenograft model in mice [8]. However, in the present study we did not observe a reduction in peritumoral vascular density in mice treated with ezrin inhibitor. It is plausible that ezrin knockdown targets functions that are independent of the p-T567 (pTERM) activation site (Fig. 2b) effected by ezrin inhibitor. Recently, Celik et al. have described a novel role for ezrin in the regulation of transcription factor DDX3 that is independent of ezrin's activation and membrane-localized open conformation initiated by phosphorylation of T567 site. This could suggest that decreasing migration capacity of cancer cells in the presence of ezrin inhibitor could play a more prominent role in reducing distant metastasis than contribution from alterations in peritumoral vascular density in our model. Further studies are required to examine the exact mechanism of action for NSC668394 ezrin inhibitor in metastatic cascade.

The relevance of $\mathrm{LN}$ metastasis in the progression of metastatic disease has been a subject of considerable debate, partly due to limited models to study the dynamics of cancer cell invasion and response to anticancer therapy within metastatic sites $[33,34]$. The intravital model presented in this study provides direct evidence that a subset of metastatic cells maintain their invasive capacity within LN metastases. Our observations are further supported by two recent studies on the fate of metastatic cells within LNs. Pereira et al. traced the fate of cancer cells expressing a photoconvertible protein and reported that a fraction of metastatic cells were able to invade the LN blood vessels, enter the blood circulation, and colonize the lungs in tumor-bearing mice [35]. Brown et al. also demonstrated that cancer cells microinfused into mouse afferent lymphatic vessels were able to disseminate via LN blood vessels and metastasize to the lungs without involvement of the thoracic duct [36]. Together, these observations in mouse models provide evidence that $\mathrm{LN}$ metastases can be a source of cancer cells for distant metastases and should therefore be part of the treatment protocol to prevent cancer progression and eliminate all disease from the patient. In fact, findings from a number of clinical trials support this theory by showing reduced rates of locoregional and systemic recurrence following addition of regional node radiation therapy to the standard of care in node-positive $\mathrm{BC}$ patients $[6,7]$.

Despite the emergence of new prognostic biomarkers and genomic profiling, the nodal status remains a key factor in $\mathrm{BC}$ prognosis and has critical therapeutic implications. Moreover, the incidence of lymph node-negative invasive $\mathrm{BC}$ has been on the rise due to advances in early detection technologies [37]. This fact, combined with less aggressive surgical biopsy and treatment of localized disease [38] plus the recent evidence that systemic spread is an early event in BC [39], highlights a need for novel prognostic factors for relapse and improved therapeutics to prevent spread of invasive cancer cells and occult micrometastases [40] in high-risk 
node-negative patients. The clinical evidence in our study points to ezrin as a potential independent prognostic marker for relapse in high-risk node-negative and node-positive $\mathrm{BC}$. It is tempting to speculate that high-risk $\mathrm{BC}$ patients with elevated tumor ezrin levels could benefit from adjuvant ezrin-targeted therapy. Blocking cancer cell dissemination by ezrin-targeted therapy could also be beneficial in certain neoadjuvant settings, where therapies such as surgery, chemotherapy, or radiation have been shown to induce cancer cell motility leading to higher numbers of circulating tumor cells [41]. Moreover, Karagiannis and colleagues have recently shown that neoadjuvant chemotherapy increases the risk of metastatic dissemination, despite decreasing the primary tumor size, in a mechanism driven by upregulation of actin-regulatory protein Mammalian-enabled (MENA) in cancer cells. Authors were able to reverse the chemotherapy-induced metastatic activity by knockdown of MENA [42]. Interestingly, ezrin acts as a protein kinase A anchoring protein (AKAP) in regulating the phosphorylation of MENA, and silencing of ezrin has been shown to inhibit MENA's function [43]. Finally, as a low tumor ezrin level is associated with improved DFS in node-positive and high-risk node-negative $\mathrm{BC}$, a less aggressive treatment regimen may be warranted in these patients to improve quality of life.

\section{Conclusions}

The effect of ezrin targeted therapy in reducing cancer cell motility, together with the clinical association of ezrin with increased risk of relapse, points to ezrin as an important regulator of the metastatic process in $\mathrm{BC}$. We therefore propose that tumor ezrin levels in $\mathrm{BC}$, and most likely in other cancers of epithelial origin, would act as an independent biomarker in predicting relapse and recommend further development of therapeutic approaches to target ezrin in patients with high tumor ezrin levels.

\section{Additional files}

Additional file 1: Figure S1. Ezrin expression in the Southeastern

Additional file 2: Figure S2. Experimental design and treatment regimen (TIFF $2020 \mathrm{~kb}$ )

Additional file 3: Video 1. Migration characteristics of GFP-EO771 ${ }^{\text {LMV }}$ cells in vitro (MOV $9130 \mathrm{~kb}$ )

Additional file 4: Video 2. Ezrin inhibitor reduces migration of cancer cells in vitro (MOV $9838 \mathrm{~kb}$ )

Additional file 5: Figure S3. Ezrin inhibitor has no effect on migration of ezrin-deficient cells (TIFF $1731 \mathrm{~kb}$ )

Additional file 6: Video 3. Intravital imaging reveals a subset of metastatic cancer cells that retain their migratory phenotype within the TDLN (MOV $2706 \mathrm{~kb}$ )

Additional file 7: Video 4. Ezrin inhibition blocks the migration of metastatic cancer cells within the TDLN (MOV $12837 \mathrm{~kb}$ )
Additional file 8: Figure S4. Ezrin knockdown in primary tumor reduces axillary $L N$ and lung metastasis in mice (TIFF 2009 kb)

\section{Acknowledgments}

The authors thank Colleen Schick for assisting with technical aspects of this study. They would also like to thank Matt Gordon at Queen's University Biomedical Imaging Centre (QUBIC) for technical assistance in flow cytometry and imaging. TMA construction and staining were performed at the Queen's Laboratory of Molecular Pathology (QLMP) by Lee Boudreau and Shakeel Virk.

\section{Funding}

This work was funded by the Cancer Research Society (19139, to BEE), the Canadian Institutes of Health Research Postdoctoral Fellowship (114518, to $A G$ ), the Canadian Breast Cancer Foundation Postdoctoral (to AG) and Graduate (to VH) Fellowships, and the Terry Fox Foundation Training Program in Transdisciplinary Cancer Research in partnership with CIHR (to $\mathrm{VH})$.

\section{Availability of data and materials}

The data generated from our breast cancer cohort (SEOBC) and corresponding TMA are not publicly available due to patient privacy reasons, but are available for access upon reasonable request. Please contact the corresponding author (AG) for further information.

\section{Authors' contributions}

$\mathrm{AG}$ and $\mathrm{VH}$ are the main drivers of the design and implementation of this study and are considered co-first authors. GT, SV, and SSG are pathologists who contributed to breast cancer TMA design, construction, and scoring. YM is an oncologist who provided clinical outcome data for our breast cancer cohort. JM assisted with intravital imaging. PAG and GM established the EO771 metastatic variant cell line and syngeneic tumor model. FK is the author of the lymphatic reporter transgenic mouse used in this study. AGD provide statistical analysis support. BEE is the corresponding author and the lead in the design and planning of this study. All authors read and approved the final manuscript.

Ethics approval and consent to participate

Written informed consent was obtained from all patients and the studies were conducted in accordance with the Queen's University Research Ethics Board consent guidelines.

Consent for publication

Not applicable.

\section{Competing interests}

The authors declare that they have no competing interests.

\section{Publisher's Note}

Springer Nature remains neutral with regard to jurisdictional claims in published maps and institutional affiliations.

\section{Author details}

${ }^{1}$ Department of Pathology and Molecular Medicine, Queen's University, Kingston, Canada. ${ }^{2}$ Max Planck Institute for Molecular Biomedicine, Münster, Germany. ${ }^{3}$ Department of Oncology, Queen's University, Kingston, Canada.

${ }^{4}$ Cancer Research Institute, Division of Cancer Biology and Genetics, Queen's University, 18 Stuart Street, Kingston, ON K7L 3N6, Canada. ${ }^{5}$ Kingston General Hospital Research Institute, Kingston, Canada.

Received: 24 August 2017 Accepted: 12 November 2018

Published online: 24 January 2019

\section{References}

1. Li J, Wei K, Yu H, Jin D, Wang G, Yu B. Prognostic Value of Ezrin in Various Cancers: A Systematic Review and Updated Meta-analysis. Sci Rep. 2015;5:17903.

2. Fehon RG, McClatchey Al, Bretscher A. Organizing the cell cortex: the role of ERM proteins. Nat Rev Mol Cell Biol. 2010;11(4):276-87.

3. Clucas J, Valderrama F. ERM proteins in cancer progression. J Cell Sci. 2014; 127(Pt 2):267-75. 
4. Padera TP, Meijer EF, Munn LL. The Lymphatic System in Disease Processes and Cancer Progression. Annu Rev Biomed Eng. 2016;18:125-58.

5. Ran S, Volk L, Hall K, Flister MJ. Lymphangiogenesis and lymphatic metastasis in breast cancer. Pathophysiology. 2010;17(4):229-51.

6. Ragaz J, Jackson SM, Le N, Plenderleith IH, Spinelli JJ, Basco VE, Wilson KS, Knowling MA, Coppin CM, Paradis M, et al. Adjuvant radiotherapy and chemotherapy in node-positive premenopausal women with breast cancer. N Engl J Med. 1997;337(14):956-62.

7. Whelan TJ, Olivotto IA, Levine MN. Regional Nodal Irradiation in Early-Stage Breast Cancer. N Engl J Med. 2015;373(19):1878-9.

8. Ghaffari A, Hoskin V, Szeto A, Hum M, Liaghati N, Nakatsu K, LeBrun D, Madarnas Y, Sengupta S, Elliott BE. A novel role for ezrin in breast cancer angio/lymphangiogenesis. Breast Cancer Res. 2014;16(5):438.

9. Yu Z, Sun M, Jin F, Xiao Q, He M, Wu H, Ren J, Zhao L, Zhao H, Yao W, et al. Combined expression of ezrin and E-cadherin is associated with lymph node metastasis and poor prognosis in breast cancer. Oncol Rep. 2015; 34(1):165-74.

10. Piao J, Liu S, Xu Y, Wang C, Lin Z, Qin Y, Liu S. Ezrin protein overexpression predicts the poor prognosis of pancreatic ductal adenocarcinomas. Exp Mol Pathol. 2015;98(1):1-6.

11. Jin T, Jin J, Li X, Zhang S, Choi YH, Piao Y, Shen X, Lin Z. Prognostic implications of ezrin and phosphorylated ezrin expression in non-small cell lung cancer. BMC Cancer. 2014;14:191.

12. Bulut G, Hong SH, Chen K, Beauchamp EM, Rahim S, Kosturko GW, Glasgow E, Dakshanamurthy S, Lee HS, Daar l, et al. Small molecule inhibitors of ezrin inhibit the invasive phenotype of osteosarcoma cells. Oncogene. 2012;31(3):269-81.

13. Celik H, Sajwan KP, Selvanathan SP, Marsh BJ, Pai AV, Kont YS, Han J, Minas TZ, Rahim S, Erkizan HV, et al. Ezrin Binds to DEAD-Box RNA Helicase DDX3 and Regulates Its Function and Protein Level. Mol Cell Biol. 2015;35(18):3145-62.

14. Paige M, Kosturko G, Bulut G, Miessau M, Rahim S, Toretsky JA, Brown ML, Uren A. Design, synthesis and biological evaluation of ezrin inhibitors targeting metastatic osteosarcoma. Bioorg Med Chem. 2014;22(1):478-87.

15. Cass JD, Varma S, Day AG, Sangrar W, Rajput AB, Raptis LH, Squire J, Madarnas Y, Sengupta SK, Elliott BE. Automated Quantitative Analysis of p53, Cyclin D1, Ki67 and pERK Expression in Breast Carcinoma Does Not Differ from Expert Pathologist Scoring and Correlates with ClinicoPathological Characteristics. Cancers (Basel). 2012;4(3):725-42.

16. McShane LM, Altman DG, Sauerbrei W, Taube SE, Gion M, Clark GM, Statistics Subcommittee of NCIEWGoCD. REporting recommendations for tumor MARKer prognostic studies (REMARK). Breast Cancer Res Treat. 2006; 100(2):229-35.

17. Sugiura K, Stock CC. Studies in a tumor spectrum. I. Comparison of the action of methylbis (2-chloroethyl)amine and 3-bis(2-

chloroethyl)aminomethyl-4-methoxymethyl -5-hydroxy-6-methylpyridine on the growth of a variety of mouse and rat tumors. Cancer. 1952;5(2):382-402.

18. Ewens A, Mihich E, Ehrke MJ. Distant metastasis from subcutaneously grown E0771 medullary breast adenocarcinoma. Anticancer Res. 2005;25(6B):3905-15.

19. Ager El, Kozin SV, Kirkpatrick ND, Seano G, Kodack DP, Askoxylakis V, Huang Y, Goel S, Snuderl M, Muzikansky A, et al. Blockade of MMP14 activity in murine breast carcinomas: implications for macrophages, vessels, and radiotherapy. J Natl Cancer Inst. 2015;107(4).

20. Hoskin V, Szeto A, Ghaffari A, Greer PA, Cote GP, Elliott BE. Ezrin regulates focal adhesion and invadopodia dynamics by altering calpain activity to promote breast cancer cell invasion. Mol Biol Cell. 2015;26(19):3464-79.

21. Gorelik R, Gautreau A. Quantitative and unbiased analysis of directional persistence in cell migration. Nat Protoc. 2014;9(8):1931-43.

22. Hagerling R, Pollmann C, Kremer L, Andresen V, Kiefer F. Intravital twophoton microscopy of lymphatic vessel development and function using a transgenic Prox1 promoter-directed mOrange2 reporter mouse. Biochem Soc Trans. 2011;39(6):1674-81.

23. Wang J, Kubes P. A Reservoir of Mature Cavity Macrophages that Can Rapidly Invade Visceral Organs to Affect Tissue Repair. Cell. 2016;165(3):668-78.

24. Srivastava J, Elliott BE, Louvard D, Arpin M. Src-dependent ezrin phosphorylation in adhesion-mediated signaling. Mol Biol Cell. 2005; 16(3):1481-90.

25. Prag S, Parsons M, Keppler MD, Ameer-Beg SM, Barber P, Hunt J, Beavil AJ, Calvert R, Arpin M, Vojnovic B, et al. Activated ezrin promotes cell migration through recruitment of the GEF Dbl to lipid rafts and preferential downstream activation of Cdc42. Mol Biol Cell. 2007;18(8):2935-48.
26. Elliott BE, Qiao H, Louvard D, Arpin M. Co-operative effect of c-Src and ezrin in deregulation of cell-cell contacts and scattering of mammary carcinoma cells. J Cell Biochem. 2004;92(1):16-28.

27. Naba A, Reverdy C, Louvard D, Arpin M. Spatial recruitment and activation of the Fes kinase by ezrin promotes HGF-induced cell scattering. EMBO J. 2008;27(1):38-50.

28. Yu Y, Khan J, Khanna C, Helman L, Meltzer PS, Merlino G. Expression profiling identifies the cytoskeletal organizer ezrin and the developmental homeoprotein Six-1 as key metastatic regulators. Nat Med. 2004;10(2):175-81.

29. Mak H, Naba A, Varma S, Schick C, Day A, SenGupta SK, Arpin M, Elliott BE. Ezrin phosphorylation on tyrosine 477 regulates invasion and metastasis of breast cancer cells. BMC Cancer. 2012;12:82.

30. Ren L, Hong SH, Cassavaugh J, Osborne T, Chou AJ, Kim SY, Gorlick R, Hewitt $\mathrm{SM}$, Khanna C. The actin-cytoskeleton linker protein ezrin is regulated during osteosarcoma metastasis by PKC. Oncogene. 2009;28(6):792-802.

31. Massague J, Obenauf AC. Metastatic colonization by circulating tumour cells. Nature. 2016:529(7586):298-306.

32. Sahai E. Illuminating the metastatic process. Nat Rev Cancer. 2007;7(10):737-49.

33. Giampieri S, Manning C, Hooper S, Jones L, Hill CS, Sahai E. Localized and reversible TGFbeta signalling switches breast cancer cells from cohesive to single cell motility. Nat Cell Biol. 2009;11(11):1287-96.

34. Das S, Sarrou E, Podgrabinska S, Cassella M, Mungamuri SK, Feirt N, Gordon $R$, Nagi CS, Wang Y, Entenberg D, et al. Tumor cell entry into the lymph node is controlled by CCL1 chemokine expressed by lymph node lymphatic sinuses. J Exp Med. 2013;210(8):1509-28.

35. Pereira ER, Kedrin D, Seano G, Gautier O, Meijer EFJ, Jones D, Chin SM, Kitahara S, Bouta EM, Chang J, et al. Lymph node metastases can invade local blood vessels, exit the node, and colonize distant organs in mice. Science. 2018;359(6382):1403-7.

36. Brown M, Assen FP, Leithner A, Abe J, Schachner H, Asfour G, Bago-Horvath Z, Stein JV, Uhrin P, Sixt M, et al. Lymph node blood vessels provide exit routes for metastatic tumor cell dissemination in mice. Science. 2018; 359(6382):1408-11.

37. Benson JR, Jatoi I, Keisch M, Esteva FJ, Makris A, Jordan VC. Early breast cancer. Lancet. 2009;373(9673):1463-79.

38. Voutsadakis IA, Spadafora S. Axillary lymph node management in breast cance with positive sentinel lymph node biopsy. World J Clin Oncol. 2015;6(1):1-6.

39. Husemann Y, Geigl JB, Schubert F, Musiani P, Meyer M, Burghart E, Forni G, Eils $R$, Fehm T, Riethmuller $G$, et al. Systemic spread is an early step in breast cancer. Cancer Cell. 2008;13(1):58-68.

40. Weaver DL, Ashikaga T, Krag DN, Skelly JM, Anderson SJ, Harlow SP, Julian TB, Mamounas EP, Wolmark N. Effect of occult metastases on survival in node-negative breast cancer. N Engl J Med. 2011;364(5):412-21.

41. Martin OA, Anderson RL, Narayan K, MacManus MP. Does the mobilization of circulating tumour cells during cancer therapy cause metastasis? Nat Rev Clin Oncol. 2017;14(1):32-44.

42. Karagiannis GS, Pastoriza JM, Wang Y, Harney AS, Entenberg D, Pignatelli J, Sharma VP, Xue EA, Cheng E, D'Alfonso TM, et al. Neoadjuvant chemotherapy induces breast cancer metastasis through a TMEM-mediated mechanism. Sci Transl Med. 2017;9(397).

43. Deming PB, Campbell SL, Stone JB, Rivard RL, Mercier AL, Howe AK. Anchoring of protein kinase A by ERM (ezrin-radixin-moesin) proteins is required for proper netrin signaling through DCC (deleted in colorectal cancer). J Biol Chem. 2015;290(9):5783-96.

Ready to submit your research? Choose BMC and benefit from:

- fast, convenient online submission

- thorough peer review by experienced researchers in your field

- rapid publication on acceptance

- support for research data, including large and complex data types

- gold Open Access which fosters wider collaboration and increased citations

- maximum visibility for your research: over $100 \mathrm{M}$ website views per year

At $\mathrm{BMC}$, research is always in progress.

Learn more biomedcentral.com/submission 